Research Article

\title{
Rational Spectral Collocation Combined with the Singularity Separated Method for a System of Singularly Perturbed Boundary Value Problems
}

\author{
Lufeng Yang $\mathbb{D}^{1,2}$ \\ ${ }^{1}$ School of Mathematics and Statistics, Lanzhou University, Lanzhou 730000, China \\ ${ }^{2}$ School of Mathematics and Information Science, North Minzu University, Yinchuan 750021, China \\ Correspondence should be addressed to Lufeng Yang; ylf-sd@163.com
}

Received 16 September 2019; Accepted 23 October 2019; Published 14 November 2019

Academic Editor: Richard I. Avery

Copyright (c) 2019 Lufeng Yang. This is an open access article distributed under the Creative Commons Attribution License, which permits unrestricted use, distribution, and reproduction in any medium, provided the original work is properly cited.

A novel rational spectral collocation method is presented combined with the singularity-separated technique for a system of singularly perturbed boundary value problems. The solution is expressed as $u=w+v$, where $w$ is the solution of the corresponding auxiliary boundary value problem and $v$ is a singular correction with explicit expressions. The rational spectral collocation method in barycentric form with the sinh transformation is applied to solve the auxiliary third boundary problem. The parameters of the singular correction can be determined by the boundary conditions of the original problem. Numerical experiments are carried out to support theoretical results and provide a favorable comparison with research results of other work.

\section{Introduction}

The singular perturbation problems (SPPs) such as fluid mechanics boundary layers, quantum mechanics turning points, and flow of large Reynolds numbers arise in the mathematical modeling physical and engineering problems. In the past decades, singular perturbation has received extensive attention. Singular perturbation boundary value problems have steep gradients in the narrow layers, which was a serious obstacle in the calculation of classical numerical methods. There is a vast literature dealing with SPPs with smooth coefficients and source term for single equation [1-3]. Compared with single equation singularly perturbed problems, coupled systems can simulate more complicated physical phenomena. Only a few authors have developed numerical methods to deal with the problem of coupled singular perturbation system on smooth data. A finite difference method on a piecewise uniform mesh for the reaction-diffusion type was proposed [4]. The result of Shishkin's method in terms of convergence, stability, and error estimation can be found in Madden and Stynes [5], Linss and Madden [6, 7], and Matthews et al. [8]. An upwinding finite difference scheme on piecewise-uniform Shishkin meshes for the convection-diffusion type was presented, and it was solved by Jacobi iteration to compute the solution [9].

In this paper, a coupled system of $m \geq 2$ singularly perturbed linear equations are considered in the unknown vector function $\mathbf{u}=\left(u_{1}, \ldots, u_{m}\right)^{T}$ :

$$
L \mathbf{u}:=-\varepsilon u^{\prime \prime}-B u^{\prime}+A u=f, \quad x \in \Omega=(0,1),
$$

and it yields

$$
\begin{aligned}
& \mathbf{u}(0)=\mathbf{b}_{0}, \\
& \mathbf{u}(1)=\mathbf{b}_{1},
\end{aligned}
$$

where $\mathbf{A}=\left(a_{i j}\right)$ and $\mathbf{B}=\left(b_{i j}\right)$ are $m \times m$ matrices and $0<\varepsilon \ll 1$ is a small parameter whose presence makes the problem singularly perturbed. We assumed that $\mathbf{f}=\left(f_{1}, \ldots, f_{m}\right)^{T} \in C^{2}[0,1]^{m}$ and both $\mathbf{b}_{0}=\left(b_{01}, \ldots, b_{0 m}\right)^{T}$ and $\mathbf{b}_{1}=\left(b_{11}, \ldots, b_{1 m}\right)^{T}$ are constant vectors. These layer behaviors can be examined in two different cases:

Case $1 . \mathbf{B} \equiv 0$, the system is called reaction-diffusion type. Generally speaking, the solution of this type has 
two boundary layers with width $O(\sqrt{\varepsilon})$ at $x=0$ and $x=1$ under some assumptions.

Case $2 . \mathbf{B} \neq 0$, the system is called convection-diffusion type, the solution of this type has a single boundary layer with width $O(\varepsilon)$ at $x=0($ or $x=1)$ under proper assumptions.

If (1) is coupled through its convective terms, we say it is strongly coupled; otherwise, if $\mathbf{B} \equiv 0$ or $\mathbf{B}$ is just a nonzero diagonal matrix, it is said to be weakly coupled.

The rational spectral collocation method was proposed in the literature [10]. A conformal map which maps the collocation points clustered near the poles of $[-1,1]$ into a new set of collocation points. The parameters of the mapping are determined by the position and width of the boundary layer. Chen and Wang [11, 12] applied a rational spectral collocation in barycentric form with the sinh transform (RSCAT) method to solve a coupled system of singularly perturbed problems and third-order singularly perturbed problems.

To weaken the singularity and improve the accuracy of numerical simulation, the singularity-separated technique (SST) for singular perturbation problem with constant coefficients was proposed by Chen and Yang [13], and finite element methods with SST were used to solve a singular perturbation problem with a single boundary layer.

Here, we present a novel numerical method based on rational spectral collocation in barycentric form with the singularly-separated method (RSC-SSM) to solve singularly perturbed boundary value problem in various types, both weakly coupled and strongly coupled.

This paper is organized as follows. The asymptotic analysis of coupled system is outlined in Section 2. The algorithmic details of the RSC-SSM for a coupled system of singularly perturbed problems are provided and the error estimates for the method are discussed in Section 3. The coupled system of singularly perturbed problems is solved in Section 4, which supports theoretical results and provides a favorable comparison with existing methods. Finally, we present some concluding remarks in Section 5.

\section{Preliminaries}

For the construction of the RSC-SSM, it is necessary to understand the properties of exact solution, especially the position and width of the boundary layer. Some useful lemmas are listed, including the maximum principle, stability result, the error estimation of the solution, and its derivatives are established for the boundary value problem (1).

2.1. Weakly Coupled System of Reaction-Diffusion Problems. If $\mathbf{A} \neq 0, \mathbf{B} \equiv 0$ in (1), it is convenient to introduce the notation to denote the reaction-diffusion operator:

$$
L_{R} \mathbf{u}:=-\varepsilon u^{\prime \prime}+\mathbf{A} \mathbf{u}=\mathbf{f} .
$$

Assume that $\mathbf{A}$ has positive diagonal entries and nonpositive off-diagonal entries, i.e.,

$$
a_{i j}= \begin{cases}>0, & i=j, \\ \leq 0, & i \neq j,\end{cases}
$$

and $\mathbf{A}$ is also strictly diagonally dominant i.e., $\sum_{\substack{k=1 \\ k \neq i}}^{m}\left|a_{i k}\right|<a_{i i}$ and $\sum_{j=1}^{m} a_{i j}>\alpha^{2}>0, i=1, \ldots, m$. These hypotheses ensure that problem (1) has a unique solution.

Lemma 1. Under above assumptions, all eigenvalues of matrix $\boldsymbol{A}$ are positive.

Proof. Let $\mathbf{A}$ have an eigenvalue of $\lambda=0$, according to the Gerschgorin disc theorem, $\exists i_{0},\left|a_{i_{0} i_{0}}\right| \leq R_{i_{0}}(\mathrm{~A})$, which is a contradiction. So, there is no zero eigenvalue in $\mathbf{A}$, that is, $|\mathbf{A}| \neq 0$.

Set $f(x)=|\mathbf{D}+x \mathbf{B}|, x \in[0,1]$, where $\mathbf{D}=\operatorname{diag}(\mathbf{A}), \mathbf{B}=$ $\mathbf{A}-\mathbf{D}$, then $f(x)$ is a continuous function on $[0,1]$ and $f(0)=|\mathbf{D}|=\prod_{i=1}^{m} a_{i i}>0$; assume $f(1)=|\mathbf{A}|<0$, then we can obtained $\exists \xi \in(0,1]$, such that $f(x)=|\mathbf{D}+\xi \mathbf{B}|=0$. Moreover, $\mathbf{A}$ is strictly diagonally dominant, then $a_{i i}>$ $R_{i}(\mathbf{A}) \geq \sum_{j=1, j \neq i}^{n}\left|\xi a_{i j}\right|$, that is, $\mathbf{D}+\xi \mathbf{B}$ is also strictly diagonally dominant. Contradictions between $f(\xi)=$ $|\mathbf{D}+\xi \mathbf{B}| \neq 0$ and $f(\xi)=0$ can be obtained. Therefore, $f(1)=|\mathbf{A}|>0$, each of the main determinants of the strictly diagonally dominant matrix is the strictly diagonally dominant, then we have the $\mathbf{A}$ is positive definite matrix. So, all eigenvalues of matrix $\mathbf{A}$ are positive.

Lemma 2. Let us suppose that a function $\mathbf{u}(x)$ satisfies $\mathbf{u}(0) \geq 0, \mathbf{u}(1) \geq 0, L \mathbf{u}(x) \leq 0, \forall x \in \Omega$, then $\mathbf{u}(x) \geq 0 ; \forall x$ $\in \bar{\Omega}$.

The direct application of the maximum principle is the following stability result.

Lemma 3. If $\mathbf{u}(x)$ is the solution of (2), then we have the stability bound inequality:

$$
\begin{aligned}
\|\mathbf{u}\| & \leq M=\max \left(\left\|\mathbf{b}_{0}\right\|,\left\|\mathbf{b}_{1}\right\|\right)+C \max \|\mathbf{f}(x)\|, \\
\left\|\mathbf{u}^{\prime}(x)\right\| & \leq \frac{C M}{\varepsilon} .
\end{aligned}
$$

Shishkin decomposition is used for asymptotic analysis of problem (3) [14], which splits the solution into regular and layer components:

$$
\mathbf{u}(x)=\mathbf{w}(x)+\mathbf{v}(x),
$$

where the regular component $\mathbf{w}(x)$ and the layer component $\mathbf{v}(x)$ are the solutions of the two problems:

$$
\begin{aligned}
& \left\{\begin{array}{l}
L_{R} \mathbf{w}=-\varepsilon \mathbf{w}^{\prime \prime}+\mathbf{A} \mathbf{w}=f, \\
\mathbf{w}(0)=\mathbf{A}^{-1} f(0), \\
\mathbf{w}(1)=\mathbf{A}^{-1} f(1),
\end{array}\right. \\
& \left\{\begin{array}{l}
L_{R} \mathbf{v}=-\varepsilon \mathbf{w}^{\prime \prime}+\mathbf{A} \mathbf{v}=\mathbf{f}, \\
\mathbf{v}(0)=\mathbf{b}_{0}-\mathbf{w}(0), \\
\mathbf{v}(1)=\mathbf{b}_{1}-\mathbf{w}(1),
\end{array}\right.
\end{aligned}
$$

then $\mathbf{w}(x)$ and $\mathbf{v}(x)$ have the following estimates. 
Lemma 4 (see [15]). Let $\mathbf{u}=\mathbf{w}+\mathbf{v}$ be the solution of problem (3) and $\mathbf{w}$ and $\mathbf{v}$ be the solutions of (7) and (8), respectively. Then, we have

$$
\begin{aligned}
& \left\|\mathbf{w}^{(j)}\right\| \leq C\left(1+\varepsilon^{(2-j / 2)}\right), \\
& \left\|\mathbf{v}^{(j)}\right\| \leq C \varepsilon^{-(j / 2)}\left(e^{-\alpha x / \sqrt{\varepsilon}}+e^{-\alpha(1-x) / \sqrt{\varepsilon}}\right),
\end{aligned}
$$

where $j=0,1,2$.

Theorem 1. The solution of (3) and (2) has the following asymptotic expansion:

$$
\mathbf{u}(x)=\mathbf{w}(x)+\mathbf{v}(\bar{x})+O(\sqrt{\varepsilon}),
$$

where

$$
\mathbf{v}(\bar{x})= \begin{cases}\mathbf{v}(x), & x \in\left[0, x_{*}\right] \cup\left[1-x_{*}, 1\right], \\ 0, \quad x \in\left[x_{*}, 1-x_{*}\right], & \\ & 0<x_{*}=-\frac{\ln \sqrt{\varepsilon}}{\alpha} \sqrt{\varepsilon} \ll 1 .\end{cases}
$$

The above theorem suggests that the boundary layer regions of the solution $\mathbf{u}(x)$ to the reaction-diffusion problems are $\left[0, x_{*}\right]$ and $\left[1-x_{*}, 1\right]$, that is, the boundary layers are located at the two endpoints of the underlying interval $[0,1]$ and each of their width is $x_{*}=-(\ln \sqrt{\varepsilon} / \alpha) \sqrt{\varepsilon}$.

2.2. Strongly Coupled System of Convection-Diffusion Problem. If $\mathbf{B} \neq 0$, the notation $L_{C}$ is introduced to denote the convection-diffusion operator:

$$
L_{C} \mathbf{u}:=-\varepsilon \mathbf{u}^{\prime \prime}-\mathbf{B} \mathbf{u}^{\prime}+\mathbf{A} \mathbf{u}=\mathbf{f} .
$$

To ensure that there is a unique solution to this problem, the following assumptions need to be satisfied: $\mathbf{B} \neq 0$ is strictly diagonally dominant and hence invertible and define $\beta=\min \left(b_{i i}\right), i=1, \ldots, m$.

The above assumption enables us to predict that the layer in $\mathbf{u}(x)$ will be at $x=0[16]$.

Theorem 2. Under above assumptions, the solution of (12) and (2) has the following asymptotic expansion:

$$
\mathbf{u}(x)=\mathbf{w}(x)+\mathbf{v}(\bar{x})+O(\varepsilon)
$$

where $\mathbf{w}(\bar{x})$ is the layer correction function, i.e.,

$$
\mathbf{v}(\bar{x})= \begin{cases}\mathbf{v}(x), & x \in\left[0, x_{*}\right], \\ 0, & x \in\left[x_{*}, 1\right]\end{cases}
$$

$$
0<x_{*}=-\frac{\ln \varepsilon}{\beta} \ll 1 .
$$

For the convection-diffusion problems, there is a boundary layer region $\left[0, x_{*}\right]$ in the solution $\mathbf{u}$ of $(12)$ and (2). That is, the boundary layers are located at the left endpoint, and its width is $x_{*}=-(\ln \varepsilon / \beta) \varepsilon$.

\section{The Singularity-Separated Technology}

Note that if $\varepsilon=10^{-k}$, then $k \geq 3, e^{-(1 / \varepsilon)}=10^{-0.43 \times 10^{k}} \leq$ $10^{-430} \approx 0$ can be neglected.

\subsection{Weakly Coupled System of Reaction-Diffusion Problem}

Theorem 3. The general solution of the homogenous equation $L_{R} \mathbf{u}=0$ can be expressed in the form

$$
\mathbf{u}=\sum_{j=1}^{m}\left[C_{j} e^{\sqrt{\lambda_{j} / \varepsilon}(x-1)}-C_{j}^{\prime} e^{-\left(\sqrt{\lambda_{j} / \varepsilon}\right) x}\right] \mathbf{V}_{j},
$$

where $\lambda_{j}$ is an eigenvalue of the $\mathbf{A}$, the vector $\mathbf{V}_{j}$ is the associated eigenvector of $\mathbf{A}$, and $C_{j}$ and $C_{j}^{\prime}$ are the constant vectors, $j=1,2, \ldots, m$.

Proof. We can write higher order differential equations as a system with a very simple change of variable:

$$
\left\{\begin{array}{l}
\mathbf{u}^{\prime}=\mathbf{v}, \\
-\varepsilon \mathbf{v}^{\prime}+\mathbf{A u}=0 .
\end{array}\right.
$$

System (16) can be written in the following matrix form:

$$
\left[\begin{array}{cc}
E_{m} & O_{m} \\
O_{m} & -\varepsilon E_{m}
\end{array}\right]\left[\begin{array}{l}
\mathbf{u} \\
\mathbf{v}
\end{array}\right]^{\prime}=\left[\begin{array}{cc}
O_{m} & E_{m} \\
-\mathbf{A} & O_{m}
\end{array}\right]\left[\begin{array}{l}
\mathbf{u} \\
\mathbf{v}
\end{array}\right] .
$$

Thus,

$$
\begin{aligned}
& {\left[\begin{array}{l}
\mathbf{u} \\
\mathbf{v}
\end{array}\right]^{\prime}=\left[\begin{array}{ll}
O_{m} & E_{m} \\
\frac{\mathbf{A}}{\varepsilon} & O_{m}
\end{array}\right]\left[\begin{array}{l}
\mathbf{u} \\
\mathbf{v}
\end{array}\right],} \\
& \text { Let } \mathbf{D}=\left[\begin{array}{ll}
O_{m} & E_{m} \\
\frac{\mathbf{A}}{\varepsilon} & O_{m}
\end{array}\right] .
\end{aligned}
$$

The eigenvalue $\lambda$ of $\mathbf{D}$ can be found from the auxiliary equation:

$$
\left|\lambda E_{2 m}-\mathbf{D}\right|=\left|\begin{array}{cc}
\lambda E_{m} & -E_{m} \\
-\frac{\mathbf{A}}{\varepsilon} & \lambda E_{m}
\end{array}\right|=0 \text {. }
$$

If $\lambda=0$, we have $\left|\lambda E_{2 m}-\mathbf{D}\right|=\left((-1)^{m^{2}} / \varepsilon^{m}\right)|\mathbf{A}| \neq 0$. So, $\lambda=0$ is not the eigenvalue of $\mathbf{D}$, and using the general transform, we can obtain

$$
\left|\begin{array}{cc}
\lambda E_{m} & -E_{m} \\
-\frac{\mathbf{A}}{\varepsilon} & \lambda E_{m}
\end{array}\right|=\left|\begin{array}{cc}
\lambda E_{m} & O_{m} \\
-\frac{A}{\varepsilon} & \lambda E_{m}-\frac{A}{\lambda \varepsilon}
\end{array}\right|=\left|\lambda E_{m}-\frac{A}{\lambda \varepsilon}\right|=0,
$$

therefore, we can get

$$
\lambda^{2}=\frac{\lambda_{j}}{\varepsilon}, \quad j=1,2, \ldots, m .
$$


where $\lambda_{j}$ is an eigenvalue of $\mathbf{A}$. The eigenvalue of matrix $\mathbf{D}$ is the square root of $\lambda_{j}$, so we can obtain all the eigenvalues of matrix D:

$$
\begin{aligned}
s_{j} & =\frac{\sqrt{\lambda}_{j}}{\sqrt{\varepsilon}}, \\
s_{j}^{\prime} & =-\frac{\sqrt{\lambda}_{j}}{\sqrt{\varepsilon}}, \\
j & =1,2, \ldots, m .
\end{aligned}
$$

Next, we need to show that $\varphi_{j}(x)=e^{s_{j}(x-1)} V_{j}$ and $\psi_{j}(x)=e^{-s_{j} x} V_{j}$ are the solutions of equation $L_{R} \mathbf{u}=0$ :

$$
\begin{aligned}
L_{R} \varphi_{j} & =-\varepsilon \varphi_{j}^{\prime \prime}(x)+A \varphi_{j}(x) \\
& =-\varepsilon\left(s_{j}\right)^{2} e^{s_{j}(x-1)} V_{j}+A e^{s_{j}(x-1)} V_{j} \\
& =\left(-\lambda_{j} V_{j}+A V_{j}\right) e^{s_{j}(x-1)} \\
& =0,
\end{aligned}
$$

that is, $\varphi_{j}(x)$ is the solution of equation $L_{R} \mathbf{u}=0$. Similarly, it can be proved that $\psi_{j}(x)$ is the solution of equation $L_{R} \mathbf{u}=0$.

Therefore, the general solution of the homogenous equation $L_{R} \mathbf{u}=0$ can be expressed in the form (15).

Corollary 1. The general solution of (3) can be expressed as follows:

$$
\mathbf{u}=\mathbf{w}+\sum_{j=1}^{m}\left[C_{j} e^{\sqrt{\lambda_{j} / \varepsilon}(x-1)}-C_{j}^{\prime} e^{-\left(\sqrt{\lambda_{j} / \varepsilon}\right) x}\right] \mathbf{V}_{j},
$$

where $\mathbf{w}$ is a special solution, $\lambda_{j}$ is an eigenvalue of $\mathbf{A}$, the vector $\mathbf{V}_{j}$ is the associated eigenvector of $\mathbf{A}$, and $C_{j}$ and $C_{j}^{\prime}$ are the constant vectors, $j=1,2, \ldots, m$.

So, the solution $\mathbf{u}(x)$ is composed of two parts, $\mathbf{u} \approx \mathbf{u}_{s s}=\mathbf{w}+\mathbf{v}$, in which $\mathbf{w}(x)$ is the regular term and $\mathbf{v}(x)$ is the singular term, and it has the following explicit expression:

$$
\mathbf{v}=\sum_{j=1}^{m}\left(C_{j} \phi_{j}(x)+C_{j}^{\prime} \psi_{j}(x)\right)
$$

The parameters $C_{j}$ and $C_{j}^{\prime}, j=1,2, \ldots, m$, in (25) can be defined by the boundary conditions. For any $\mathbf{f}(\mathbf{x})$, how to construct the regular solution $\mathbf{w}$ is key.

We construct an auxiliary third boundary value problem to solve the special solution of (3):

$$
\left\{\begin{array}{l}
L \mathbf{w}=-\varepsilon w^{\prime \prime}+\mathbf{A} \mathbf{w}=\mathbf{f}(x), \\
\mathbf{A w}(0)=\mathbf{f}(0), \\
\mathbf{A w}(1)=\mathbf{f}(1),
\end{array}\right.
$$

then $\varepsilon \mathbf{w}^{\prime \prime}(0)=0, \varepsilon \mathbf{w}^{\prime \prime}(1)=0$, and the singularities of the solution are weakened. Based on the boundary condition (2), we can obtain the undetermined coefficients $C_{j}$ and $C_{j}^{\prime}$ in explicit singular function (15):

$$
\left\{\begin{array}{l}
\sum_{j=0}^{m} C_{j}^{\prime} \mathbf{V}_{j}=\mathbf{b}_{0}-\mathbf{w}(0), \\
\sum_{j=0}^{m} C_{j} \mathbf{V}_{j}=\mathbf{b}_{1}-\mathbf{w}(1) .
\end{array}\right.
$$

Due to the linear independence of $\left\{\mathbf{V}_{j}\right\}_{j=1}^{m}$, the matrix $\mathbf{V}=\left[\mathbf{V}_{1}, \mathbf{V}_{2}, \ldots, \mathbf{V}_{m}\right]$ is invertible. Equation (27) has a unique solution.

\subsection{Strongly Coupled System of Convection-Diffusion Problem}

Theorem 4. If $A \equiv 0$, the general solution of (12) can be expressed as follows:

$$
\mathbf{u}=\mathbf{w}+\sum_{j=1}^{m} C_{j} e^{-\left(\lambda_{j} / \varepsilon\right) x} \mathbf{V}_{j}
$$

where $\mathbf{w}$ is a special solution, $\lambda_{j}$ and $\mathbf{V}_{j}$ are the distinct eigenvalues and eigenvectors of $\mathbf{B}$, and $C_{j}$ is the constant which is determined by boundary condition (2).

Proof. Let $\mathbf{u}^{\prime}=\mathbf{v}$, then (12) can be transformed into an equivalent problem including a system of two ODEs as follows:

$$
\left\{\begin{array}{l}
\mathbf{u}^{\prime}=\mathbf{v}, \\
-\varepsilon \mathbf{v}^{\prime}-\mathbf{B v}=0 .
\end{array}\right.
$$

System (29) can be written in the following matrix form:

$$
\left[\begin{array}{cc}
E_{m} & O_{m} \\
O_{m} & -\varepsilon E_{m}
\end{array}\right]\left[\begin{array}{l}
\mathbf{u} \\
\mathbf{v}
\end{array}\right]^{\prime}=\left[\begin{array}{cc}
O_{m} & E_{m} \\
O_{m} & \mathbf{B}
\end{array}\right]\left[\begin{array}{l}
\mathbf{u} \\
\mathbf{v}
\end{array}\right],
$$

where $E_{m}$ and $O_{m}$ are identity matrix and all-zeros matrix, respectively. Then, we have

$$
\begin{aligned}
& {\left[\begin{array}{l}
\mathbf{u} \\
\mathbf{v}
\end{array}\right]^{\prime}=\left[\begin{array}{ll}
O_{m} & E_{m} \\
O_{m} & \frac{-\mathbf{B}}{\varepsilon}
\end{array}\right]\left[\begin{array}{l}
\mathbf{u} \\
\mathbf{v}
\end{array}\right],} \\
& \operatorname{set} \mathbf{D}=\left[\begin{array}{ll}
O_{m} & E_{m} \\
O_{m} & \frac{-\mathbf{B}}{\varepsilon}
\end{array}\right] .
\end{aligned}
$$

Let $\lambda$ is an eigenvalue of $\mathbf{D}$, and

$$
\left|\lambda E_{2 m}-\mathbf{D}\right|=\left|\begin{array}{cc}
\lambda E_{m} & -E_{m} \\
O_{m} & \lambda E_{m}+\frac{\mathbf{B}}{\varepsilon}
\end{array}\right|=0 .
$$

The eigenvalue $\lambda=s_{0}=0$ has multiplicity $m$, $\lambda=s_{j}=-\left(\lambda_{j} / \varepsilon\right),(j=1,2, \ldots, m)$.

Next, we need to show that $\varphi_{j}(x)=e^{-\left(\lambda_{j} / \varepsilon\right)} V_{j}$ is the solution of equation $L_{C} \mathbf{u}=0$ :

$$
\begin{aligned}
L_{C} \varphi_{j} & =-\varepsilon \varphi_{j}^{\prime \prime}(x)-B \varphi_{j}^{\prime}(x) \\
& =-\frac{\lambda_{j}^{2}}{\varepsilon} e^{-\left(\lambda_{j} / \varepsilon\right) x} V_{j}+B \frac{\lambda_{j}}{\varepsilon} e^{-\left(\lambda_{j} / \varepsilon\right) x} V_{j} \\
& =\left(-\lambda_{j} V_{j}+B V_{j}\right) \frac{\lambda_{j}}{\varepsilon} e^{-\left(\lambda_{j} / \varepsilon\right) x}
\end{aligned}
$$$$
=0 \text {. }
$$ 
So, the general solution of (12) can be expressed in the form (28).

Let the singular correction function be

$$
\mathbf{v}(x)=\sum_{j=1}^{m} C_{j} e^{-\left(\lambda_{j} / \varepsilon\right) x} \mathbf{V}_{j} .
$$

In the same way, the regular term $\mathbf{w}$ is a solution of an auxiliary third boundary value problem:

$$
\left\{\begin{array}{l}
L \mathbf{w}=-\varepsilon w^{\prime \prime}+\mathbf{B} \mathbf{w}^{\prime}=\mathbf{f}(x), \\
\mathbf{B} \mathbf{w}^{\prime}(0)=\mathbf{f}(0) \\
\mathbf{w}(1)=\mathbf{b}_{1}
\end{array}\right.
$$

According to the boundary conditions, we can obtain the undetermined coefficients in an explicit singular function:

$$
\sum_{j=1}^{m} C_{j}^{\prime} \mathbf{V}_{j}=\mathbf{b}_{0}-\mathbf{w}(0) \text {. }
$$

\section{The Rational Spectral Collocation Method Combined with Singularity-Separated Method}

4.1. The Barycentric Form of Rational Interpolation. Rational function $p_{N}(x)$ in barycentric form, which interpolates function $u(x)$ at $N+1$ distinct points $\left\{x_{k}\right\}_{k=0}^{N}$ can be expressed as [17]

$$
u(x) \approx p_{N}(x)=\frac{\sum_{k=0}^{N}\left(\omega_{k} / x-x_{k}\right) u\left(x_{k}\right)}{\sum_{k=0}^{N} \omega_{k} / x-x_{k}},
$$

where $\left\{\omega_{k}\right\}_{k=0}^{N}$ are barycentric weights. For ChebyshevGauss-Lobatto points $x_{k}=-\cos (k \pi / N)$, barycentric weights are chosen as [17]

$$
\begin{aligned}
\omega_{0} & =\frac{1}{2}, \\
\omega_{k} & =(-1)^{k}, \\
k & =1,2, \ldots, N-1, \\
\omega_{N} & =\frac{(-1)^{N}}{2} .
\end{aligned}
$$

According to the theorem in Baltensperger et al. [18], we have the convergence analysis of the rational interpolation polynomial in barycentric form with transformed Chebyshev points as follows:

$$
\left\|p_{N}(x)-u(x)\right\|=O\left(\rho^{-N}\right) \cdot \forall x \in[-1,1],
$$

where $p_{N}(x)$ is the rational interpolating polynomial in barycentric form of $u, g$ is a conformal map, and $\rho>1$ is the sum of its major and minor axes of an ellipse.

An advantage of the rational interpolation in barycentric form is that its derivatives can be calculated directly using differentiation formulae, instead of using the differential quotient rule repeatedly. The $n$th order derivative of polynomial $p_{N}(x)$ evaluated at the point $x_{j}$, and can be expressed in the form

$$
\begin{aligned}
p_{N}^{(n)}\left(x_{j}\right) & =\sum_{k=0}^{N} \frac{d^{n}}{d x^{n}}\left(\frac{\left(\omega_{k} / x-x_{k}\right) u\left(x_{k}\right)}{\sum_{l=0}^{N} \omega_{l} / x-x_{l}}\right)_{x=x_{j}} \\
& =\sum_{k=0}^{N} D_{j k}^{(n)} u\left(x_{k}\right), \quad j=0,1, \ldots, N,
\end{aligned}
$$

where $D_{j k}^{(n)}$ is the entry of the $n$th order differentiation matrix. The entries of the first- and second-order differentiation matrices, are given by [17]

$$
\begin{aligned}
& D_{j k}^{(1)}=\left\{\begin{array}{l}
\frac{\omega_{k}}{\omega_{j}\left(x_{j}-x_{k}\right)}, \quad j \neq k, \\
-\sum_{i \neq k} D_{j i}^{(1)}, \quad j=k,
\end{array}\right. \\
& D_{j k}^{(2)}=\left\{\begin{array}{l}
2 D_{j k}^{(1)}\left(D_{j j}^{(1)}-\frac{1}{x_{j}-x_{k}}\right), \quad j \neq k . \\
-\sum_{i \neq k} D_{j i}^{(2)}, \quad j=k .
\end{array}\right.
\end{aligned}
$$

As suggested in (39), the convergence rate of the rational spectral collocation method mainly depends on the analytic region of $u$ in the complex plane. Therefore, the conformal map $g$ could be chosen to enlarge the ellipse of analyticity of $u \circ g$. Thus, compared with the Chebyshev spectral method, a better approximation of $u$ could be obtained.

Note that differentiation matrices (41) only rely on weights $\omega_{k}$ and new points $\widehat{x}_{k}$, which is why the underlying equation does not need to be converted to new coordinates after maps.

4.2. The sinh Transform. In order to approximate the rapid changes in the boundary layer region. Tee and Trefethen have constructed the conformal map [19]:

$$
\begin{aligned}
g(x)= & \lambda+\mu \sinh \left[\left(\sinh ^{-1}\left(\frac{1-\lambda}{\mu}\right)+\sinh ^{-1}\left(\frac{1+\lambda}{\mu}\right)\right) \frac{x-1}{2}\right. \\
& \left.+\sinh ^{-1}\left(\frac{1-\lambda}{\mu}\right)\right],
\end{aligned}
$$

where $\lambda$ and $\mu$ are the location and width of the boundary layers, respectively. The transformed Chebyshev points $\left\{g_{\lambda, \mu}\left(x_{k}\right)\right\}_{k=0}^{N}$ are clustered near the location of boundary layer $x=\lambda$, and the density is determined by the width of boundary layer. For the convection-diffusion type, parameters in (42) should be chosen as follows:

$$
\begin{aligned}
& \lambda=-1 \\
& \mu=-2 \frac{\ln \varepsilon}{\beta} \varepsilon .
\end{aligned}
$$


In order to better distinguish the singular perturbation problem with two boundary layers, Tee proposed the combined sinh transform as

$$
\tilde{g}_{\mu}(x)= \begin{cases}\frac{1}{2}\left[g_{-1, \mu}(2 x+1)-1\right], & x \in[-1,0), \\ \frac{1}{2}\left[g_{1, \mu}(2 x-1)+1\right], & x \in[0,1] .\end{cases}
$$

All derivatives of the piecewise map $\tilde{g}$ at $x=0$ are continuous so that the spectral accuracy could be preserved. For the reaction-difffusion type, the parameter in (44) should be chosen as $\mu=-2(\ln \sqrt{\varepsilon} / \alpha) \sqrt{\varepsilon}$.

4.3. RSC-SSM. In this part we will show the RSC-SSM in a detailed algorithm.

For reaction-diffusion, we solve auxiliary third boundary value problem (26) using a rational spectral collocation method with the sinh transformation. First, by introducing the transformation $x=(y+1) / 2$ and defining $\widehat{u}(y)=u(x)=u((y+1) / 2)$, then $u^{\prime}(x)=2 \widehat{u}^{\prime}(y)$ and $u^{\prime \prime}$ $(x)=4 \widehat{u}^{\prime \prime}(y)$. Evaluating equations in (26) at points $y_{k}, k=$ $0,1, \ldots, N$, yields

$$
\left(-4 \varepsilon \mathbf{E}_{\mathbf{m}} \otimes \mathbf{D}^{(2)}+\mathbf{A} \otimes \mathbf{E}_{m}\right) \mathbf{W}=\mathbf{F},
$$

where

$$
\begin{aligned}
& \mathbf{W}=\left[\begin{array}{c}
\widehat{w}_{1}(\mathbf{Y}) \\
\vdots \\
\widehat{w}_{m}(\mathbf{Y})
\end{array}\right], \\
& \widehat{w}_{i}(\mathbf{Y})=\left[\begin{array}{c}
\widehat{w}_{i}\left(y_{0}\right) \\
\vdots \\
\widehat{w}_{i}\left(y_{N}\right)
\end{array}\right], \\
& \mathbf{F}=\left[\begin{array}{c}
\widehat{f}_{1}(\mathbf{Y}) \\
\vdots \\
\widehat{f}_{m}(\mathbf{Y})
\end{array}\right], \\
& \widehat{f}_{i}(\mathbf{Y})=\left[\begin{array}{c}
\hat{f}_{i}\left(y_{0}\right) \\
\vdots \\
\hat{f}_{i}\left(y_{N}\right)
\end{array}\right],
\end{aligned}
$$

for $i=1, \ldots, m, \otimes$ is Kronecker product.

Boundary conditions in (26) suggest that

$$
\begin{aligned}
\left(\mathbf{A} \otimes \mathbf{E}_{m}(1)\right) \mathbf{W} & =\hat{f}_{i}\left(y_{0}\right), \\
\left(\mathbf{A} \otimes \mathbf{E}_{m}(N+1)\right) \mathbf{W} & =\widehat{f}_{i}\left(y_{N}\right), \\
i & =1,2, \ldots, m .
\end{aligned}
$$

Solving the linear algebra system composed of (45) and (47), we can obtain the numerical solution of (26), which has a boundary layer which occurs at $y=1$ with the width $\mu=2 x_{*}$. Let the transformed Chebyshev collocation points be

$$
Y=\left\{y_{k}\right\}_{k=0}^{N}=\left\{\tilde{g}_{\mu}\left(-\cos \left(\frac{k \pi}{N}\right)\right)\right\}_{k=0}^{N},
$$

where $\widetilde{g}_{\mu}$ is expressed by (44) in which $\mu=2 x_{*}$.

As for the convection-diffusion type, evaluating equations in (35) at points $y_{k}, k=0,1, \ldots, N$, yields

$$
\left(-4 \varepsilon \mathbf{E}_{m} \otimes \mathbf{D}^{(2)}-2 \mathbf{B} \otimes \mathbf{D}^{(1)}\right) \mathbf{W}=\mathbf{F} .
$$

Boundary conditions in (35) suggest that

$$
\begin{aligned}
\left(-2 \mathbf{B} \otimes \mathbf{D}^{(1)}(1)\right) \mathbf{W} & =\widehat{f}_{i}\left(y_{0}\right), \\
\widehat{w}_{i}\left(y_{N}\right) & =b_{1 i}, \\
i & =1,2, \ldots, m .
\end{aligned}
$$

Solving the linear algebra system composed of (49) and (50), we can obtain the numerical solution of (35).

The numerical solution of original problem (1) and (2) can be obtained as $u^{N}=W+V$.

\subsection{Errors Analysis}

Theorem 5. Let $\mathbf{u}\left(x_{i}\right)$ and $\mathbf{u}^{N}\left(x_{i}\right)$ be the exact solution and numerical solution of the original problem (3) and (2), respectively. Then, we have

$$
\left\|\mathbf{u}-\mathbf{u}^{N}\right\| \leq c_{1} e^{-\left(\sqrt{\varrho_{A}} / \sqrt{\varepsilon}\right)}+c_{2} \rho^{-N},
$$

where $\varrho_{A}$ is the spectral radius of $\mathbf{A}$.

Proof. Let $\mathbf{z}=\mathbf{u}-\mathbf{u}^{N}$, then the error $\mathbf{z}$ satisfies

$$
\|\mathbf{z}\|=\left\|\mathbf{u}-\mathbf{u}^{N}\right\|=\left\|\mathbf{u}-\mathbf{u}_{s}+\mathbf{u}_{s}-\mathbf{u}^{N}\right\| \leq\left\|\mathbf{u}-\mathbf{u}_{s}\right\|+\left\|\mathbf{w}+\mathbf{v}-\mathbf{u}^{N}\right\| .
$$

According to the proof of Theorem 5, the singular correct function $\mathbf{v}(x)$ is the solution of the homogeneous equations $L \mathbf{v}=0$. So, set $\mathbf{R}=\mathbf{u}-\mathbf{u}_{S S}$ :

$$
L\left(\mathbf{u}-\mathbf{u}_{S}\right)=L(\mathbf{u}-\mathbf{w}-\mathbf{v})=-\varepsilon \mathbf{u}^{\prime \prime}+A \mathbf{u}-\left(-\varepsilon \mathbf{w}^{\prime \prime}+A \mathbf{u}\right)-L \mathbf{v}=0 .
$$

Simultaneously, obtained from (27)

$$
\begin{aligned}
& \mathbf{R}(0)=\mathbf{u}(0)-\mathbf{w}(0)-\sum_{j=1}^{m} C_{j}^{\prime} \mathbf{V}_{j}+\sum_{j=1}^{m} C_{j} \mathbf{V}_{j} e^{-\lambda_{j}}=\sum_{j=1}^{m} C_{j} \mathbf{V}_{j} e^{-\lambda_{j}}, \\
& \mathbf{R}(1)=\mathbf{u}(1)-\mathbf{w}(1)-\sum_{j=1}^{m} C_{j} \mathbf{V}_{j}+\sum_{j=1}^{m} C_{j}^{\prime} \mathbf{V}_{j} e^{-\lambda_{j}}=\sum_{j=1}^{m} C_{j}^{\prime} \mathbf{V}_{j} e^{-\lambda_{j}} .
\end{aligned}
$$

According to the Lemma 3, we can get

$$
\|\mathbf{R}\|=\left\|\mathbf{u}-\mathbf{u}_{s}\right\| \leq\left\|\sum_{j=1}^{m} C_{j}^{\prime} \mathbf{V}_{j} e^{-\lambda_{j}}\right\|+\left\|\sum_{j=1}^{m} C_{j} \mathbf{V}_{j} e^{-\lambda_{j}}\right\| \leq c_{1} e^{-\left(\sqrt{\varrho_{A}} / \sqrt{\varepsilon}\right)} .
$$

And, using the convergence of rational spectral collocation (39), we obtain 


$$
\left\|\mathbf{w}+\mathbf{v}-\mathbf{u}^{N}\right\| \leq c_{2} \rho^{-N} .
$$

Joint (55) and (56), we have the error estimation

$$
\left\|\mathbf{u}-\mathbf{u}^{N}\right\| \leq\left\|\mathbf{u}-\mathbf{u}_{s}\right\|+\left\|\mathbf{w}+\mathbf{v}-\mathbf{u}^{N}\right\| \leq c_{1} e^{-(\sqrt{\varrho} / \sqrt{\varepsilon})}+c_{2} \rho^{-N} .
$$

Similarly, for the convection-diffusion type, we have

$$
\left\|\mathbf{u}-\mathbf{u}^{N}\right\| \leq\left\|\mathbf{u}-\mathbf{u}_{s}\right\|+\left\|\mathbf{w}+\mathbf{v}-\mathbf{u}^{N}\right\| \leq c_{1} e^{-\left(\varrho_{B} / \varepsilon\right)}+c_{2} \rho^{-N},
$$

where $\varrho_{B}$ is the spectral radius of $\mathbf{B}$.

So, I can get the convergence of the RSC-SSM that can almost achieve spectral accuracy.

\section{Numerical Experiments}

In order to demonstrate the accuracy and efficiency of the RSC-SSM, we use RSCAT and RSC-SSM to solve singularly perturbed problems with exact solutions and compare the results. We verify the theoretical results obtained in the previous section through numerical experiments. The relative maximum errors of the solution are given by

$$
e=\frac{\left\|u_{N}-u_{E}\right\|_{\infty}}{\left\|u_{E}\right\|_{\infty}},
$$

where $u_{N}$ and $u_{E}$ are the numerical solution and the exact solution, respectively.

In our computations, all experiments are carried outperformed using MATLAB (version R2014a) on a personal computer with a $2.5 \mathrm{GHz}$ central processing unit (Intel Core i5-2450M), 4.00 GB of memory, and Windows 7 operating system.

Example 1. Consider the reaction-diffusion problem with constant coefficients:

$$
\begin{aligned}
\mathbf{B} & =0 \\
\mathbf{A} & =\left(\begin{array}{cc}
2 & -1 \\
-1 & 2
\end{array}\right), \\
\mathbf{f} & =\left(\begin{array}{l}
1 \\
2
\end{array}\right) \\
\mathbf{b}_{0} & =\mathbf{B}_{1}=\left(\begin{array}{l}
0 \\
0
\end{array}\right) .
\end{aligned}
$$

The exact solution of this problem is

$$
u=\frac{1}{2}(1,1)^{T} u_{1}+\frac{1}{2}(-1,1)^{T} u_{2}
$$

where

$$
\begin{aligned}
& u_{1}=3 \frac{e^{(x-2) / \sqrt{\varepsilon}}-e^{-x / \sqrt{\varepsilon}}-e^{(x-1) / \sqrt{\varepsilon}}+e^{-(x+1 / \sqrt{\varepsilon})}+3,}{1-e^{-2 / \sqrt{\varepsilon}}}+ \\
& u_{2}=\frac{1}{3} \frac{e^{\sqrt{3}(x-2) / \sqrt{\varepsilon}}-e^{-(\sqrt{3} x / \sqrt{\varepsilon})}-e^{\sqrt{3}(x-1) / \sqrt{\varepsilon}}+e^{-(\sqrt{3}(x+1) / \sqrt{\varepsilon})}}{1-e^{-2 \sqrt{3} / \sqrt{\varepsilon}}}+\frac{1}{3} .
\end{aligned}
$$

This problem has two boundary layer regions, i.e., the boundary layer are located at the two endpoints of the underlying interval $[0,1]$. In our method, we use the transform (44), and the parameter is chosen as $\alpha=0.99$. The eigenvalues of $\mathbf{A} / \varepsilon$ are $\lambda_{1}=1 / \varepsilon$ and $\lambda_{2}=3 / \varepsilon$, and $\mathbf{V}_{1}=(1,1)^{T}$ and $\mathbf{V}_{2}=$ $(1,-1)^{T}$ are the corresponding eigenvectors. So, the general solution of this problem can be expressed (24), and the special solution $\mathbf{w}$ is a solution of an auxiliary third boundary value problem (26). The undetermined coefficients of singular term can be obtained by (27).

Figure 1 plots the relative maximum errors of both RSCAT and RSC-SSM in semilog scale for various values of $\varepsilon$. Compared with RSCAT, the convergence rates have considerably improved in our RSC-SSM on over trend. Furthermore, Table 1 provides the comparison of numerical results obtained by our RSC-SSM and RSCAT with several choices of $\varepsilon$. We perceive that our method gives much better results with significantly fewer number of unknowns. With the increase of $N$, the relative maximum error of RSCAT method decreases, while quite the opposite is true for the RSC-SSM. Figure 2 displays plots of numerical and exact solutions with $\varepsilon=10^{-2}$ and $\varepsilon=10^{-6}$. There are more points located in the boundary layer region. Figure 3 shows the point-wise errors of the function in the whole region, it is observed that compared with RSCAT, the smaller the parameter $\varepsilon$ is, the more accurate the RSC-SSM result.

Example 2. Consider the convection-diffusion problem with constant coefficients:

$$
\begin{aligned}
& \mathbf{B}=\left(\begin{array}{ccc}
3 & -1 & -1 \\
-1 & 4 & -2 \\
-1 & -2 & 4
\end{array}\right), \\
& \mathbf{f}=\left(\begin{array}{c}
-4 \\
11 \\
-7
\end{array}\right) \\
& \mathbf{A}=0, \\
& \mathbf{b}_{0}=\left(\begin{array}{c}
-1 \\
4 \\
-1
\end{array}\right)
\end{aligned}
$$$$
\mathbf{b}_{1}=\left(\begin{array}{c}
e^{-1 / \mathcal{\varepsilon}}-2 e^{-4 / \varepsilon}+1 \\
e^{-1 / \varepsilon}+e^{-4 / \varepsilon}+2 e^{-6 / \varepsilon}-2 \\
e^{-1 / \varepsilon}+e^{-4 / \varepsilon}-2 e^{-6 / \varepsilon}
\end{array}\right) \text {. }
$$

The exact solution of this problem is

$$
\begin{aligned}
\mathbf{u}= & (1,1,1)^{T} e^{-x / \varepsilon}+(-2,1,1)^{T} e^{-4 x / \varepsilon}+(0,2,-2)^{T} e^{-6 x / \varepsilon} \\
& +(x,-2 x, x-1)^{T} .
\end{aligned}
$$




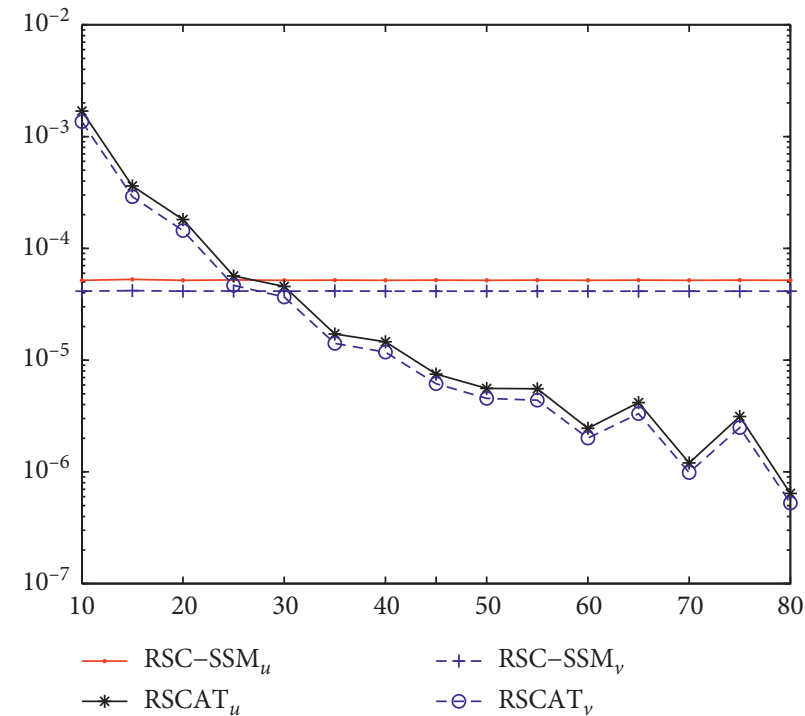

(a)

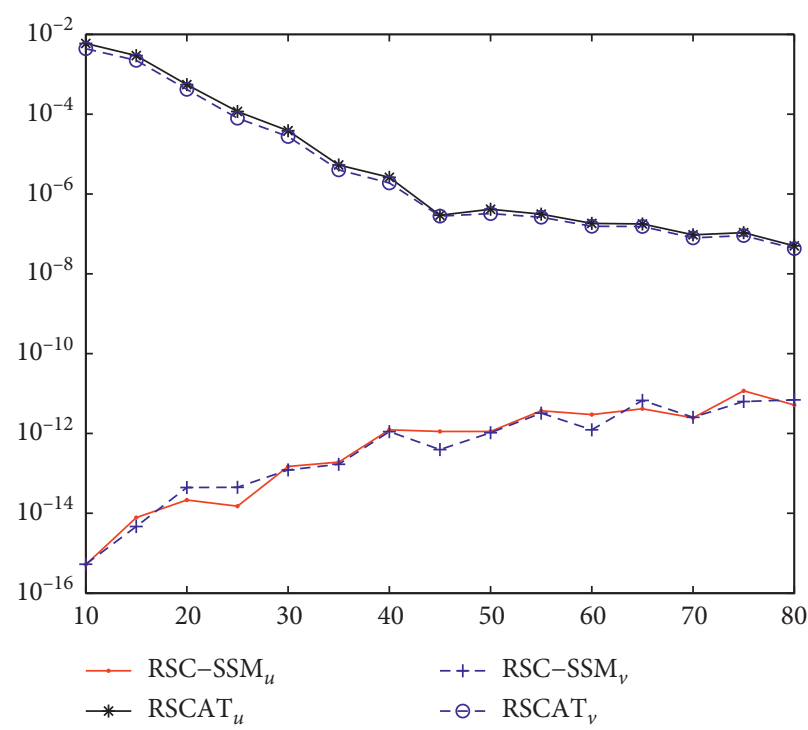

(c)

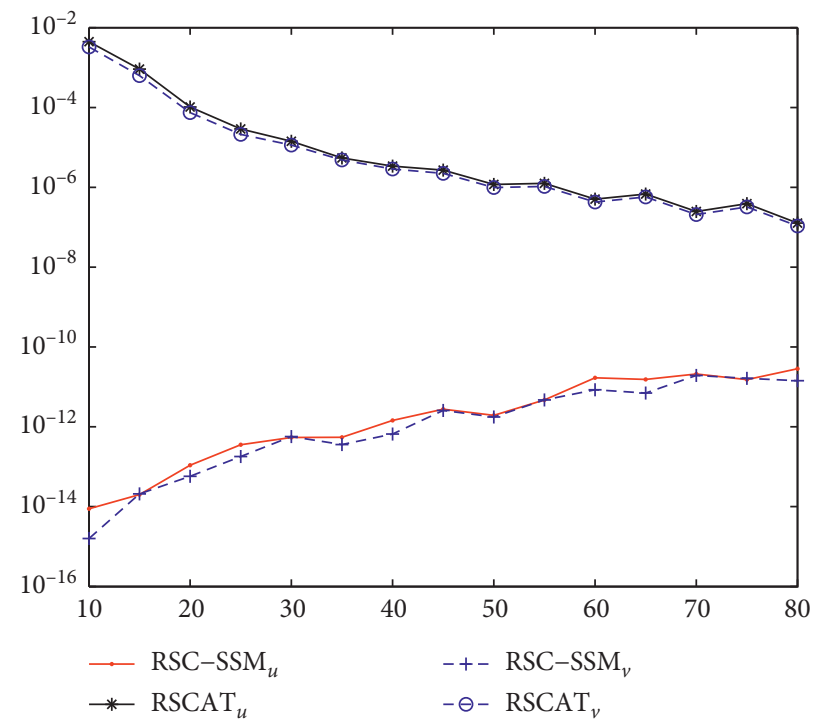

(b)

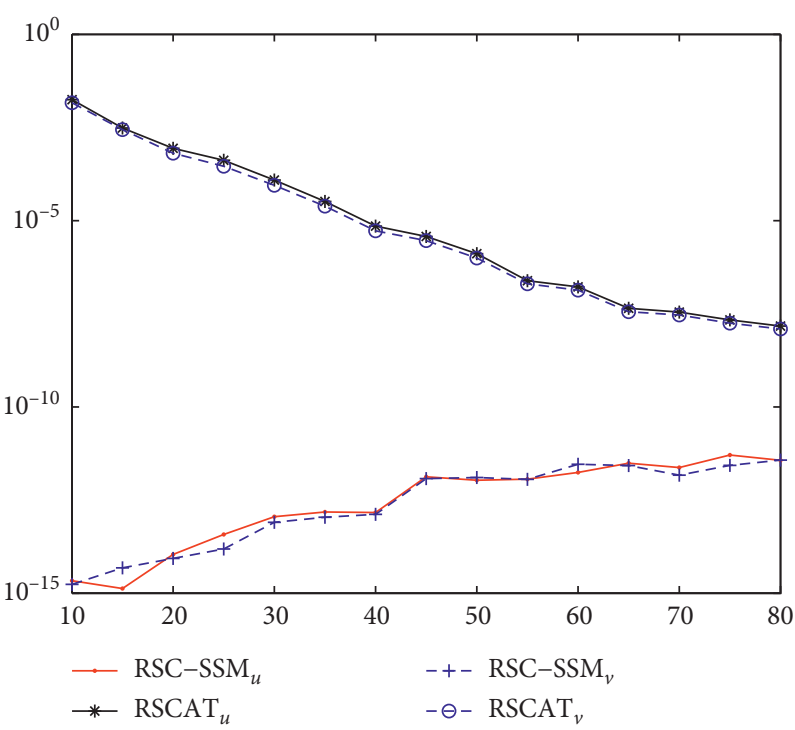

(d)

Figure 1: Relative maximum errors in Example 1. (a) $\varepsilon=10^{-1}$. (b) $\varepsilon=10^{-4}$. (c) $\varepsilon=10^{-6}$. (d) $\varepsilon=10^{-8}$.

TABle 1: Comparison of the relative maximum errors for Example 1.

\begin{tabular}{lccccc}
\hline$\varepsilon$ & $N$ & RSC-SSM & & RSCAT \\
& & $u$ & $v$ & $1.61 e-03$ & \\
\hline $1 e-2$ & 20 & $5.19 e-05$ & $4.14 e-05$ & $6.74 e-06$ & $1.29 e-03$ \\
$1 e-4$ & 40 & $2.33 e-12$ & $4.10 e-12$ & $1.05 e-07$ & $5.70 e-06$ \\
$1 e-6$ & 60 & $7.49 e-14$ & $2.04 e-13$ & $5.63 e-06$ & $1.06 e-07$ \\
$1 e-8$ & 60 & $1.50 e-15$ & $8.66 e-16$ & $4.77 e-06$ \\
\hline
\end{tabular}

For the sake of comparison, the parameters in transform (42) are chosen as $\beta=2.55$. This problem has rapid changes near the point $x=0$. The eigenvalues of $\mathbf{B} / \varepsilon$ are $\lambda_{1}=1 / \varepsilon, \lambda_{2}=4 / \varepsilon$, and $\lambda_{3}=6 / \varepsilon$, and the corresponding eigenvectors are $\quad \mathbf{V}_{1}=(1,1,1)^{T}, \mathbf{V}_{2}=(2,-1,-1)^{T}$, and $\mathbf{V}_{3}=(0,-1,1)^{T}$. So, the general solution of this problem can be expressed (28), and the special solution $\mathbf{w}$ is a solution of auxiliary third boundary value problem (35). The 


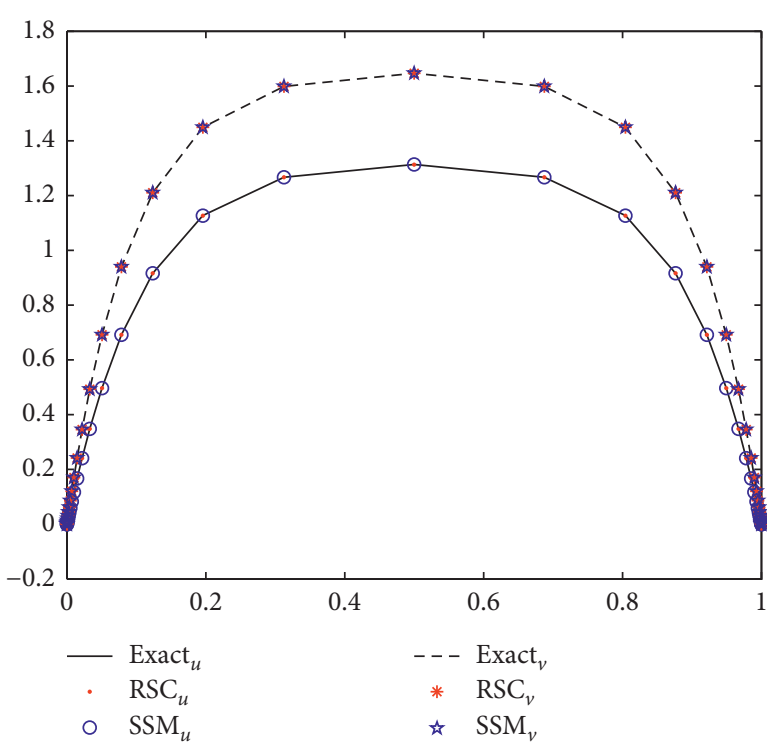

(a)

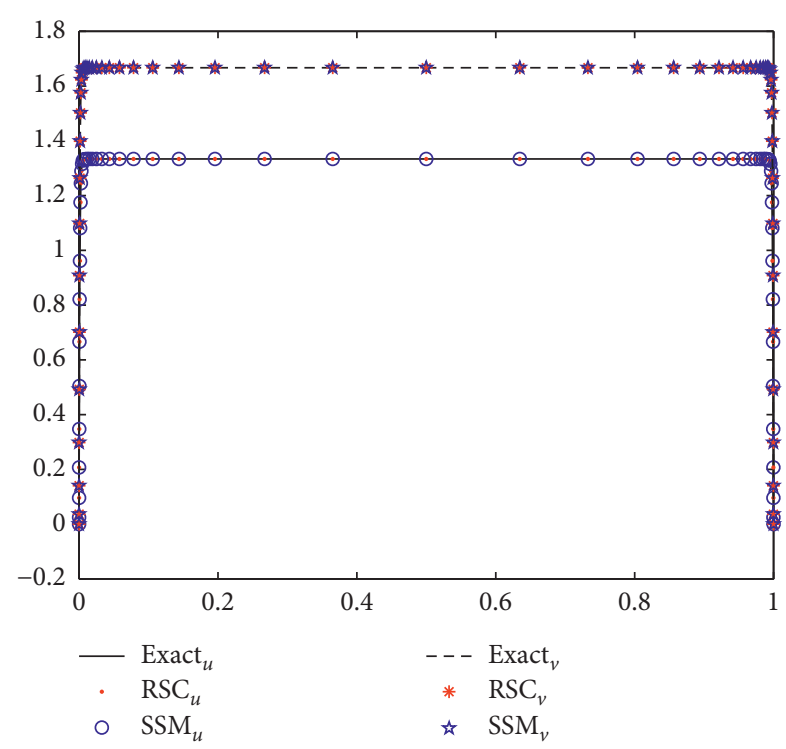

(b)

Figure 2: Numerical solutions vs. exact solutions( $u_{1}$ : solid lines and $u_{2}$ : dashed lines). (a) $\varepsilon=10^{-2}$ and $N=40$. (b) $\varepsilon=10^{-6}$ and $N=60$.

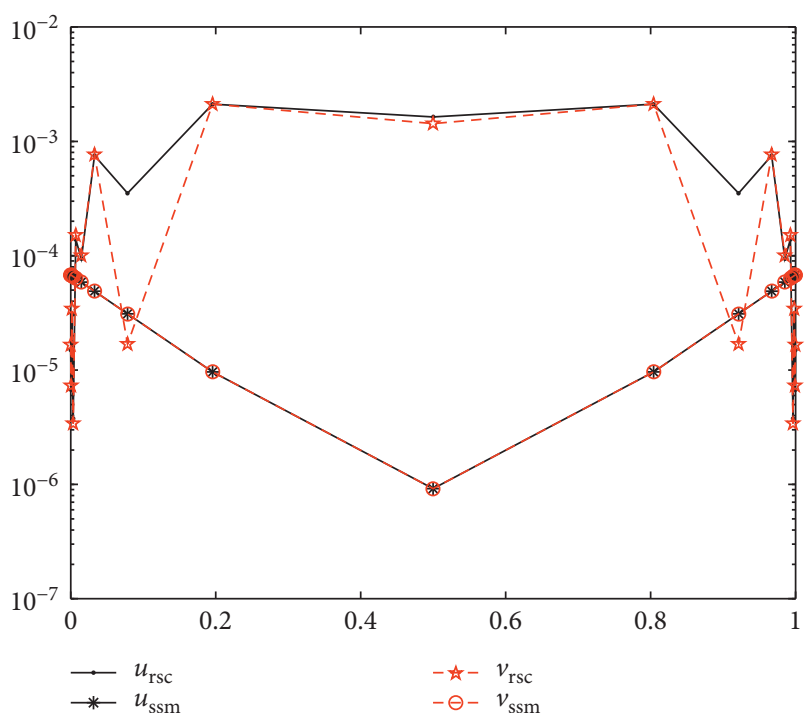

(a)

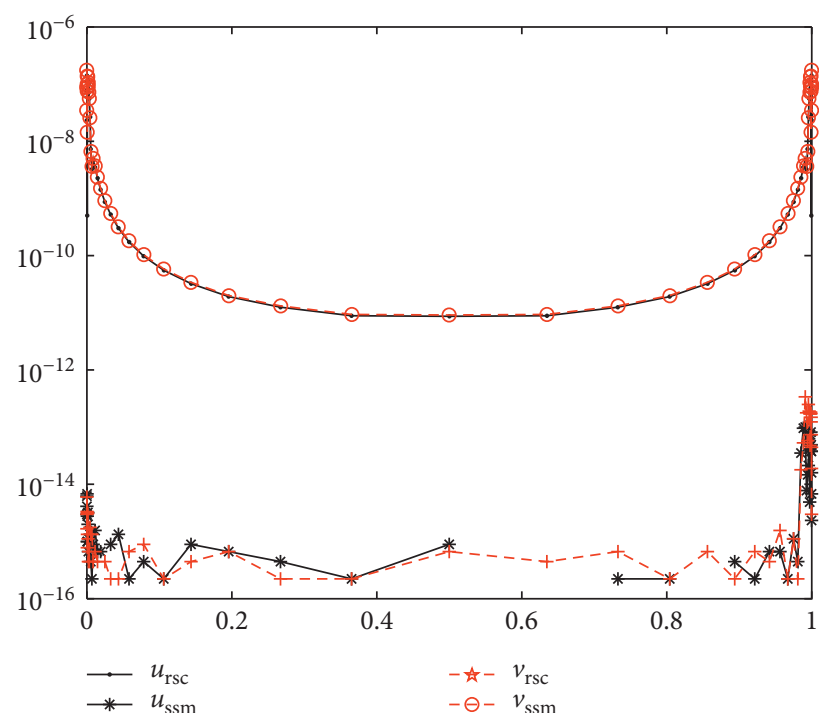

(b)

Figure 3: Pointwise relative errors in Example 1: RSCAT (dashed lines) vs. RSC-SSM (solid lines). (a) $\varepsilon=10^{-2}$ and $N=20$. (b) $\varepsilon=10^{-6}$ and $N=60$.

undetermined coefficients of singularity term can be obtained by (36). We compare the maximum relative errors of the RSC-SSM with the RSCAT method in Figure 4 and Table 2, and both verify the high accuracy and efficiency of our method.

Figure 4 plots the relative maximum errors of both RSCAT and RSC-SSM in semilog scale for various values of $\varepsilon$. It shows that the convergence rates have greatly improved in our RSC-SSM. Besides, Table 2 illustrates the comparison of numerical results obtained by our
RSC-SSM and the method in [12] with several choices of $\varepsilon$. We observe that our method gives much better results with significantly less number of unknowns. Figure 5 displays the plots of numerical and exact solutions for the case with $\varepsilon=10^{-2}$ and $\varepsilon=10^{-6}$. There are more points located in the boundary layer region. Figure 6 shows the pointwise errors of the function in the whole region; it is observed that compared with RSCAT, we can conclude that with the increase of $N$, the error of the RSC-SSM remains stable. 


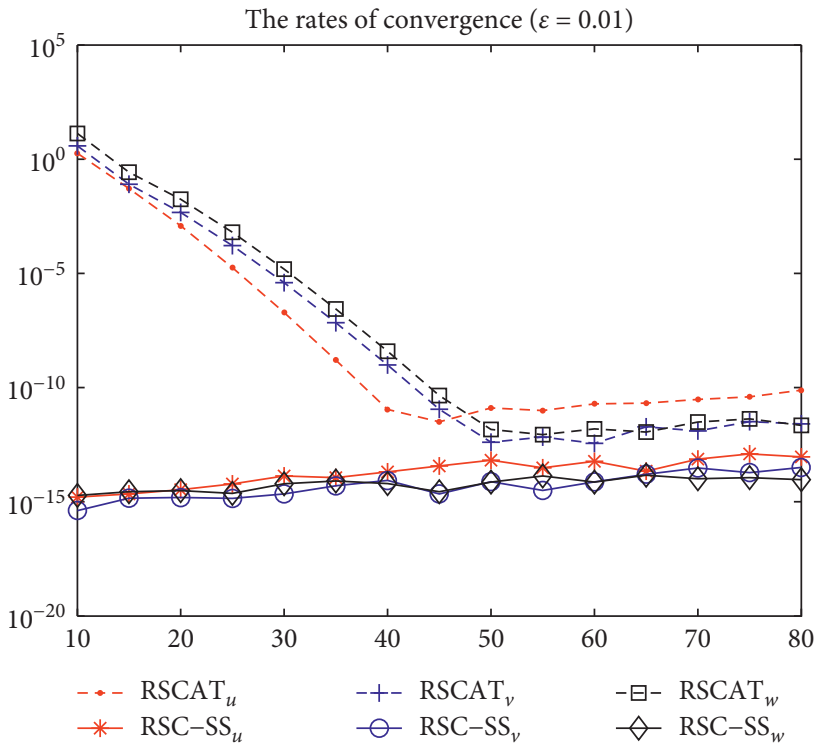

(a)

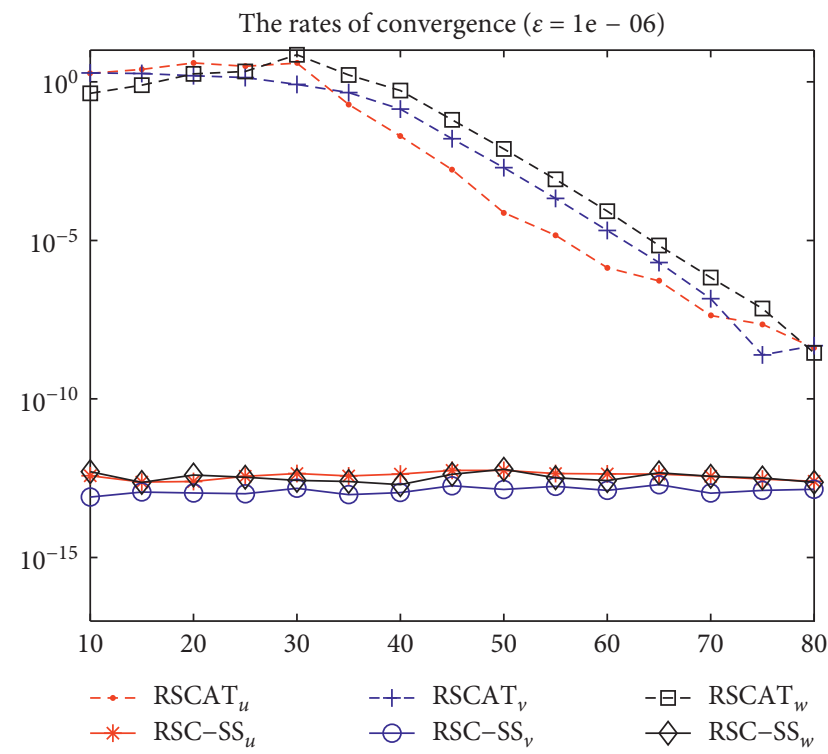

(b)

Figure 4: Relative maximum errors in Example 2: RSCAT (dashed lines) vs. RSC-SSM (solid lines). (a) $\varepsilon=10^{-2}$. (b) $\varepsilon=10^{-6}$.

TABle 2: Comparison of the relative maximum errors for Example 2.

\begin{tabular}{|c|c|c|c|c|c|c|c|}
\hline \multirow{2}{*}{$\varepsilon$} & \multirow{2}{*}{$N$} & \multicolumn{3}{|c|}{ RSC-SSM } & \multicolumn{3}{|c|}{ RSCAT } \\
\hline & & $u$ & $v$ & $w$ & u & $v$ & $w$ \\
\hline $1 e-2$ & 20 & $3.39 e-15$ & $1.55 e-15$ & $3.11 e-15$ & $1.21 e-03$ & $4.70 e-03$ & $1.76 e-02$ \\
\hline $1 e-4$ & 40 & $3.12 e-14$ & $9.77 e-15$ & $3.52 e-14$ & $1.53 e-05$ & $2.58 e-04$ & $1.01 e-03$ \\
\hline $1 e-6$ & 60 & $4.33 e-13$ & $1.31 e-13$ & $2.67 e-13$ & $1.38 e-06$ & $2.05 e-05$ & $8.35 e-05$ \\
\hline $1 e-8$ & 60 & $6.87 e-12$ & $1.23 e-12$ & $3.18 e-12$ & $3.34 e-04$ & $9.71 e-03$ & $3.81 e-02$ \\
\hline
\end{tabular}

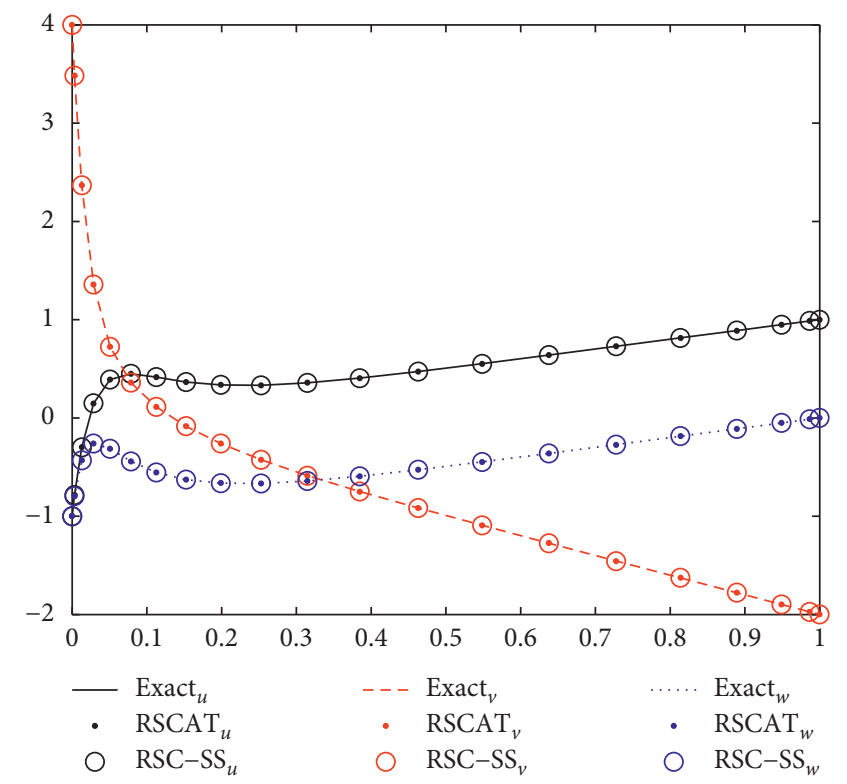

(a)

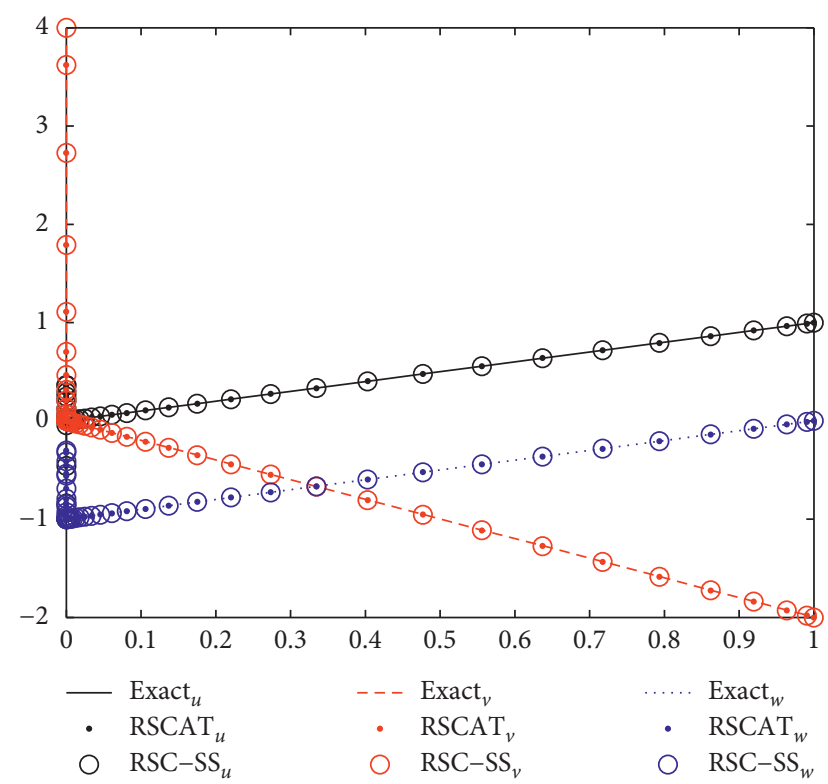

(b)

FIgURe 5: Numerical solutions vs. exact solutions. RSCAT (dashes lines) vs. RSC-SSM (solid lines). (a) $\varepsilon=10^{-2}$ and $N=20$. (b) $\varepsilon=10^{-6}$ and $N=60$. 


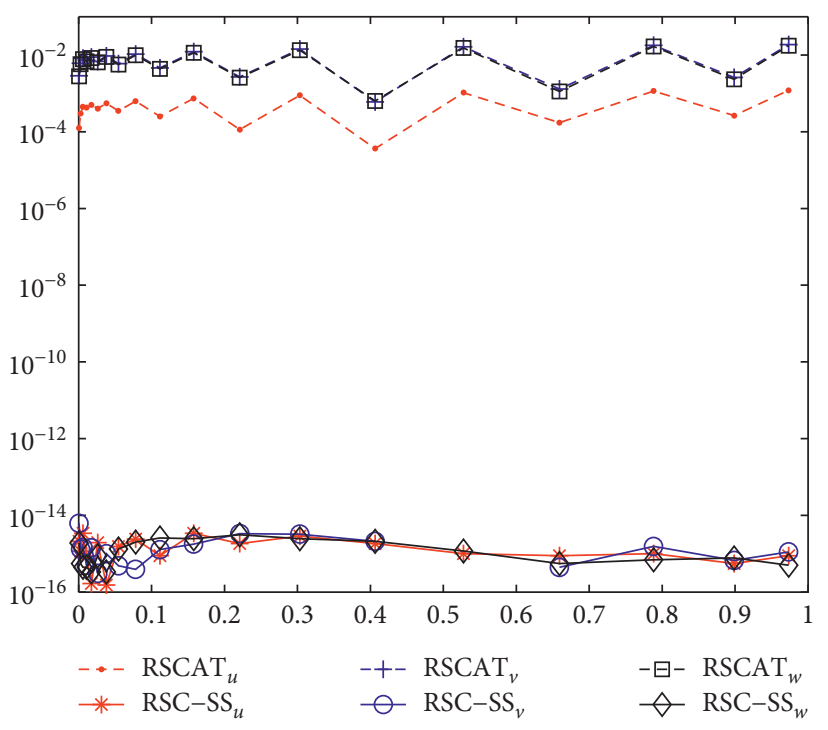

(a)

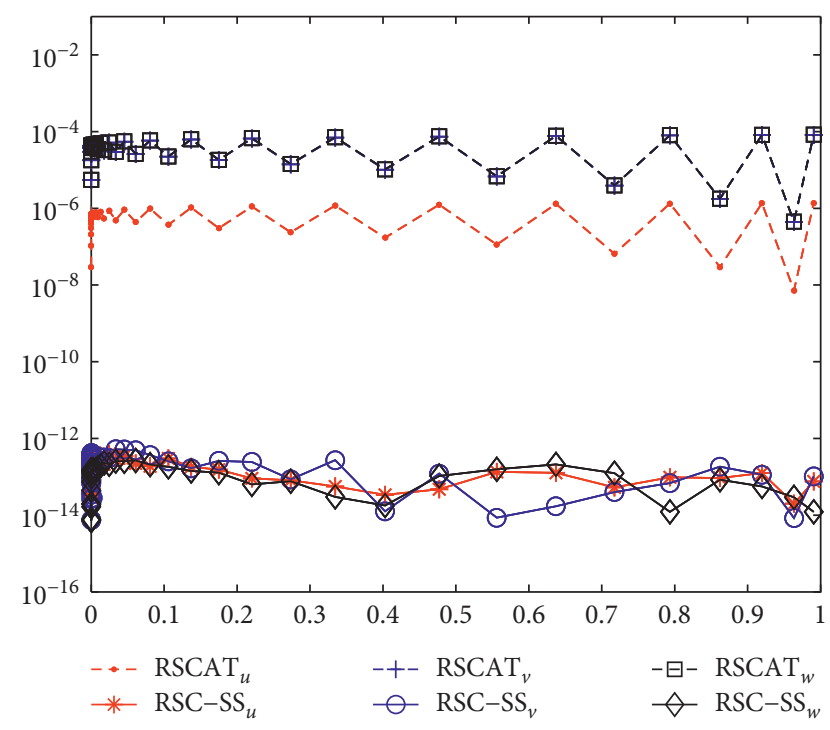

(b)

Figure 6: Pointwise errors. RSCAT (dashes lines) vs. RSC-SSM method (solid lines).

\section{Concluding Remarks}

In this paper, a new numerical method RSC-SSM has been proposed to solve a system of singularly perturbed boundary value problems. The numerical solution consists of two parts: the solution of weaker singularly auxiliary equation and the singular correction term. Numerical experiments confirm that compared to RSCAT, the present RSC-SSM has the following advantages:

(1) The RSC-SSM is easy to implement, and it enjoys computational efficiency, accuracy, and stability over some popular numerical approaches.

(2) The accuracy of numerical approximation depends not only on the number of the grid nodes, but also on parameter $\varepsilon$. The smaller the $\varepsilon$ is, the thinner the boundary layer is, and the better results obtained.

(3) In the RSC-SSM, we make good use of the eigenvalue of coefficient matrix, which plays a important role in constructing general solution of singularly perturbed problems.

The numerical results demonstrate the spectral accuracy of proposed algorithms and agree with the theoretical analysis very well. And, the theoretical and numerical frameworks presented in this paper are essential for extension to more complicated problems.

\section{Data Availability}

The data used to support the findings of this study are included within the article

\section{Conflicts of Interest}

The author declares that there are conflicts of interest.

\section{Acknowledgments}

The author is grateful to Prof. Y J Wu for his valuable suggestions and help for the work related to this paper. The work is partially supported by the First-Class Disciplines Foundation of Ningxia (No. NXYLXK2017B09), Natural Science Foundation of Ningxia (No. NZ17105), and the Science Foundation of North Minzu University (No. 2019XYZSX04).

\section{References}

[1] M. K. Kadalbajoo and K. C. Patidar, "A survey of numerical techniques for solving singularly perturbed ordinary differential equations," Applied Mathematics and Computation, vol. 130, no. 2-3, pp. 223-259, 2002.

[2] M. Kumar and N. Singh, "A collection of computational techniques for solving singular boundary-value problems," Advances in Engineering Software, vol. 40, no. 4, pp. 288-297, 2009.

[3] M. K. Kadalbajoo and V. Gupta, "A brief survey on numerical methods for solving singularly perturbed problems," Applied Mathematics and Computation, vol. 217, no. 8, pp. 3641-3716, 2010.

[4] G. I. Shishkin, "Mesh approximation of singularly perturbed boundary-value problems for system of elliptic and parabolic equations," Computational Mathematics and Mathematical Physics, vol. 35, no. 4, pp. 429-446, 1995.

[5] N. Madden and M. Stynes, "A uniformly convergent numerical method for a coupled system of two singularly perturbed linear reaction-diffusion problems," IMA Journal of Numerical Analysis, vol. 23, no. 4, pp. 627-644, 2003.

[6] T. Linss and N. Madden, "An improved error estimate for a numerical method for a system of coupled singluar perturbed reaction-diffusion equations," Computational Methods in Applied Mathematics, vol. 3, no. 3, pp. 417-423, 2003.

[7] T. Linss, N. Madden, Accurate solution of a system of coupled singularly perturbed reaction-diffusion equations," Computing, vol. 73, no. 2, pp. 121-133, 2004. 
[8] S. Matthews, E. O’Riordan, and G. I. Shishkin, "A numerical method for a system of singularly perturbed reaction-diffusion equations," Journal of Computational and Applied Mathematics, vol. 145, no. 1, pp. 151-166, 2002.

[9] E. O'Riordan, J. Stynes, and M. Stynes, “An iterative numerical algorithm for a strongly coupled system of singularly perturbed convection-diffusion problems," in Lecture Notes in Computer Science, vol. 5434, pp. 104-115, SpringerVerlag, Berlin, Germany, 2009.

[10] J.-P. Berrut, R. Baltensperger, and H. D. Mittelmann, "Recent developments in barycentric rational interpolation," Trends and Applications in Constructive Approximation, vol. 15, pp. 27-51, 2005.

[11] S. Chen and Y. Wang, "A rational spectral collocation method for third-order singularly perturbed problems," Journal of Computational and Applied Mathematics, vol. 307, pp. 93105, 2016.

[12] S. Chen, Y. Wang, and X. Wu, "Rational spectral collocation method for a coupled system of singularly perturbed boundary valus problems," Journal of Computational Mathematics, vol. 29, no. 4, pp. 458-473, 2011.

[13] C. M. Chen and J. Yang, "The singularity-separated method for the singular perturbation problems in 1-d," International Journal of Numerical Analysis and Modeling, vol. 15, no. 1-2, pp. 102-110, 2017.

[14] J. J. H. Miller, E. O'Riordan, and G. I. Shishkin, Fitted Numerical Methods for Singular Perturbation Problems-Error Estimates in the Maximum Norm for Linear Problems in One and Two Dimensions, World Scientific, Singapore, 1996.

[15] T. Linss and N. Madden, "Layer-adapted meshes for a linear system of coupled singularly perturbed reaction-diffusion problems," IMA Journal of Numerical Analysis, vol. 29, no. 1, pp. 109-125, 2009.

[16] H. G. Roos, M. Stynes, and L. Tobiska, Robust Numerical Methods for Singularly Perturbed Differential Equations, Springer-Verlag, Berlin, Germany, 1996.

[17] J.-P. Berrut and L. N. Trefethen, "Barycentric Lagrange interpolation,” SIAM Review, vol. 46, no. 3, pp. 501-517, 2004.

[18] R. Baltensperger, J.-P. Berrut, and B. Noël, "Exponential convergence of a linear rational interpolant between transformed Chebyshev points," Mathematics of Computation, vol. 68, no. 227, pp. 1109-1121, 1999.

[19] T. W. Tee and L. N. Trefethen, "A rational spectral collocation method with adaptively transformed Chebyshev grid points," SIAM Journal on Scientific Computing, vol. 28, no. 5, pp. 1798-1811, 2006. 


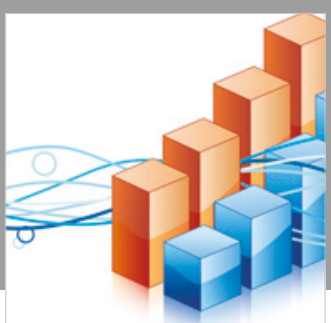

Advances in

Operations Research

\section{-n-m}
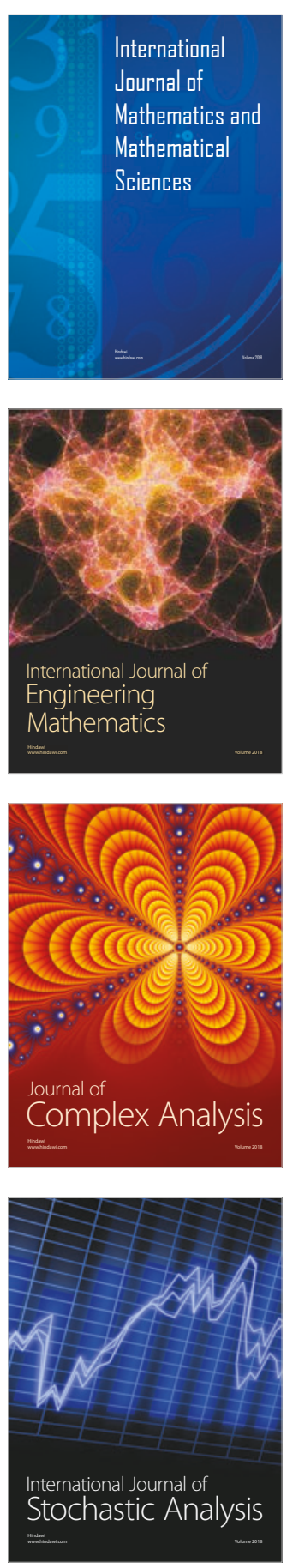
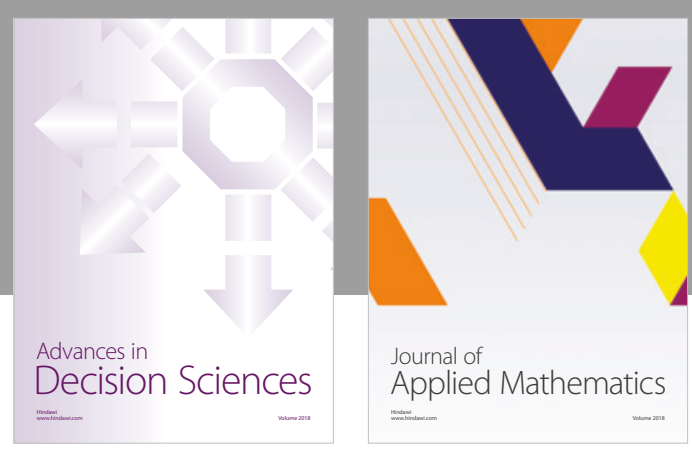

Journal of

Applied Mathematics
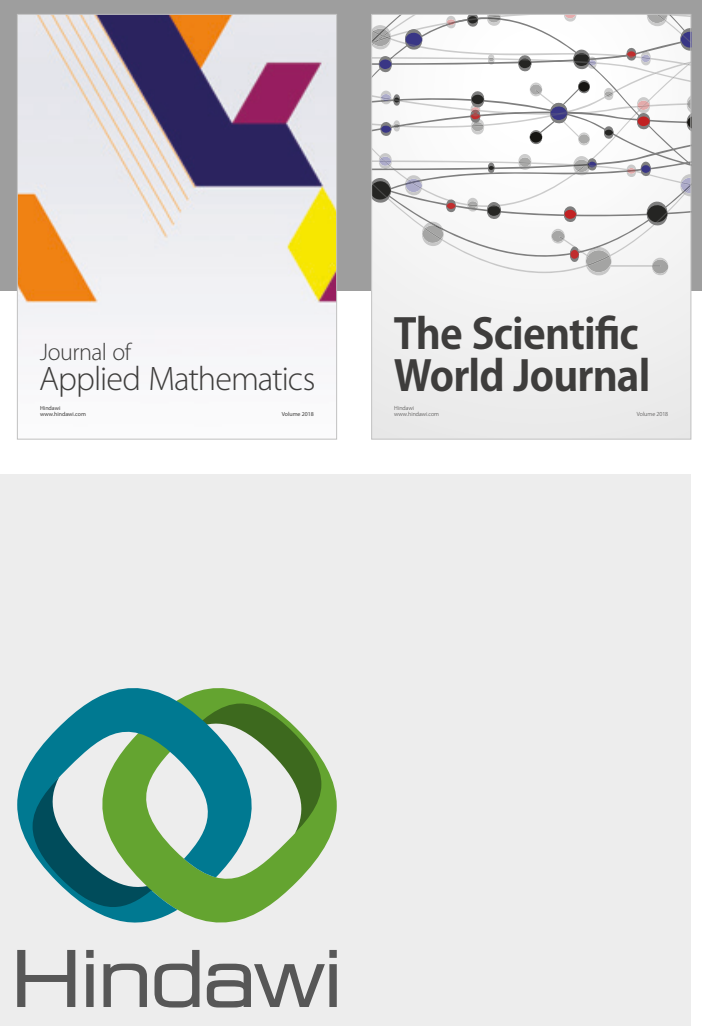

Submit your manuscripts at

www.hindawi.com

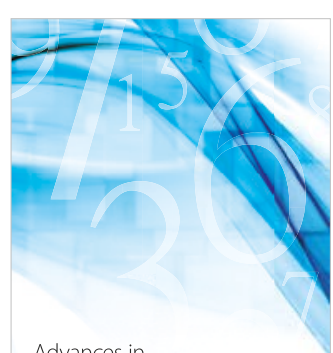

Advances in
Numerical Analysis
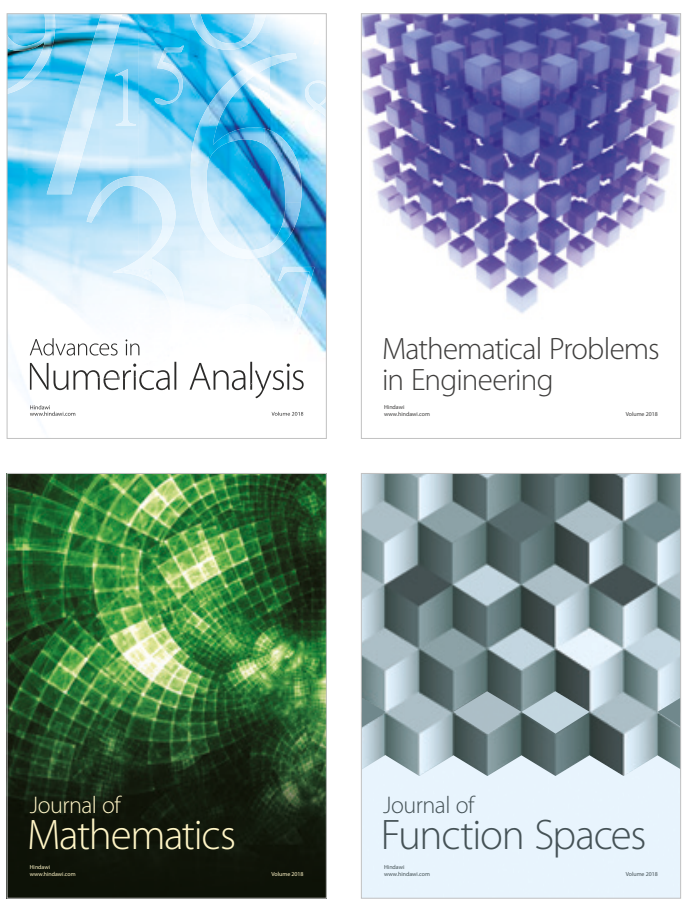

Mathematical Problems in Engineering

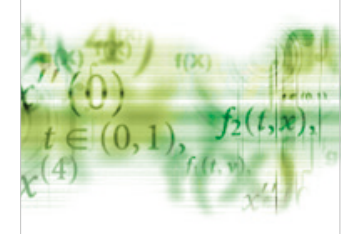

International Journal of

Differential Equations

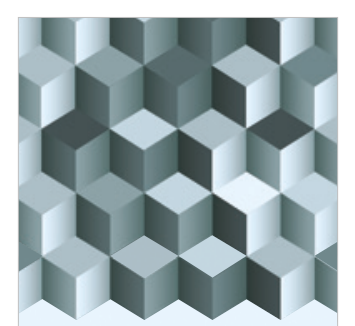

Journal of

Function Spaces

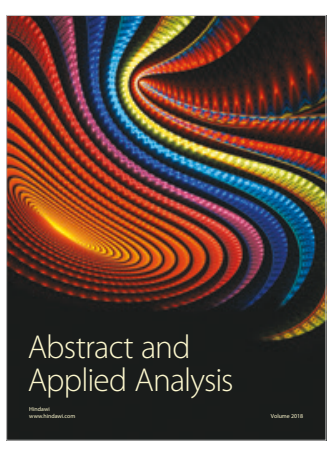

The Scientific

World Journal

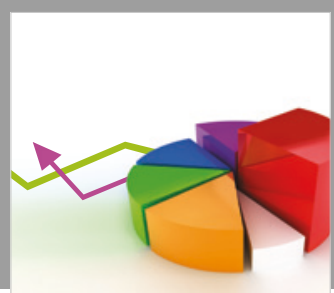

Journal of

Probability and Statistics
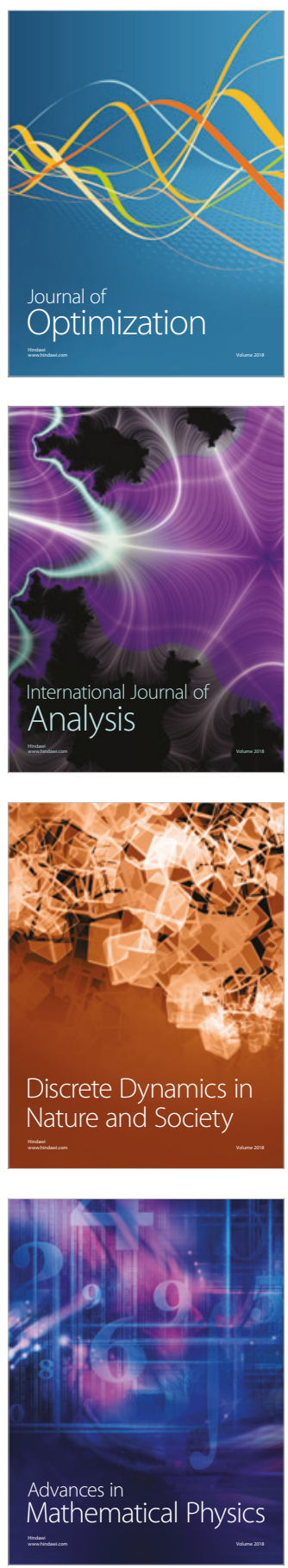